\title{
CALIDAD DE VIDA LABORAL: HISTORIA, DIMENSIONES Y BENEFICIOS
}

\author{
QUALITY OF WORKING LIFE: \\ HISTORY, DIMENSIONS AND BENEFITS \\ Isabel Granados P. ${ }^{1}$ \\ Universidad Nacional Mayor de San Marcos, Lima, Perú \\ (Recibido el 10/09/2011, ACEPtado el 2/12/ 2011)
}

\begin{abstract}
RESUMEN
El propósito de este trabajo consiste en realizar una recopilación teórica de la historia de la calidad de vida laboral, así como de los diversos conceptos que se han elaborado en torno a ella. Para ello, se realiza una revisión de los trabajos más destacados. Asimismo, se estudian los dos amplio grupos de dimensiones que se relacionan a la CVL: las condiciones objetivas y las condiciones subjetivas, se detalla cada uno de los componentes de ambas dimensiones. Por último, se lista los diversos beneficios que trae consigo la implementación de programas de CVL en la empresa.
\end{abstract}

Palabras clave: Calidad de vida laboral, motivación, satisfacción laboral.

\begin{abstract}
ABSTRAC
The purpose of this paper is to perform a theorical compilation of the history of the Quality of Working Life and the various concepts that have developed around it. In order to archive this, most relevant works are reviewed. Also, the two broad groups of dimensions that relate to QWL are examined: the objective and subjective conditions, each one of the components of both dimensions are outlined. Finally, the various benefits that come with the implementation of programs of QWL in the company are listed.
\end{abstract}

Keywords: Quality of working life, motivation, job satisfaction.

1 Estudiante del X ciclo de Psicología de la Universidad Nacional Mayor de San Marcos E-maill: isabel.granados-ext@bshg. 


\section{INTRODUCCIÓN}

En la actualidad, los dramáticos cambios que caracterizan la Nueva Economía están reestructurando el ambiente laboral. El fenómeno de la globalización ha generado un gran nivel de competencia internacional. Las corporaciones que deseen ser exitosas y consolidar su posicionamiento en el mercado deberán ser corporaciones innovadoras, diversas y flexibles; debido a que tanto los consumidores como los inversores ejercen una fuerte presión para que las empresas generen ambientes de trabajo justos, productivos y potenciadores de las capacidades de los trabajadores y los equipos de trabajo.

Nuestro país no es ajeno a los cambios de esta Nueva Economía. En los últimos 20 años, el Perú está afrontando cambios sustanciales no solo en lo relacionado al aspecto económico, sino también al político, social y cultural, como resultado de la globalización. La implementación de nuevas tecnologías tanto en la empresa como en el mercado nacional, así como un incremento en la inversión privada extranjera ha permitido este crecimiento económico en nuestro país. A pesar de ello, el crecimiento económico no garantiza el éxito de las organizaciones. Estas han tomado conciencia de que ni la tecnología ni el dinero podrán garantizarles el éxito, ni la efectividad ante las exigencias de la sociedad mientras no se revalore el principal capital de la empresa: el capital humano.

Así, se han desarrollado diversas investigaciones vinculadas a diversos factores humanos relacionados al ambiente laboral (valores, compromiso, clima, satisfacción, autoestima, entre otros) que puedan estar influyendo en el rendimiento de los trabajadores para el desarrollo efectivo de las organizaciones. Peters y R.H. Waterman (1982) estudiaron sobre los valores, encontrando que la mayoría de las características de las organizaciones exitosas se basan en los valores humanos, como prioridad de sus acciones. J. Cruz (1996) y M. Rodríguez (1993) consideran que la autoestima y los valores organizacionales son importantes en el éxito personal y organizacional. (Citado en Loli y Cuba, 2007). Es así que las empresas deben asumir una nueva responsabilidad sobre el desarrollo personal de los trabajadores. Las organizaciones se han vuelto más competitivas, se vive la ola de las empresas de calidad, de empresas eficientes, en todas ellas, los trabajadores juegan un rol importantísimo, por lo que la calidad de vida laboral juega un papel importante para tener éxito en la gestión de hoy.

En la actualidad, las principales compañías están innovando las políticas de calidad de vida en la empresa y sus prácticas para hacerse cargo de las necesidades de todos los empleados, como una forma de apoyar los objetivos del negocio y de retener a los mejores talentos.

\section{HISTORIA}

La administración científica se centraba especialmente en la especialización y eficiencia de las tareas en estructuras tradicionales de la organización. A medida que esta estructura fue evolucionando, se buscaba una división plena de las tareas, buscando sobretodo la eficiencia, reduciendo costos y usando una mano de obra no calificada que puede capacitarse en corto tiempo para el desempeño del trabajo. Las tareas a realizar eran definidas por las jerarquías al mando del personal técnico. Esta estructura presenta muchas dificultades, principalmente, dejaba de lado una adecuada calidad de vida laboral. En este contexto se 
notaron ausentismos, aburrimientos por las tareas repetitivas, alta rotación de personal y la calidad sufrió un descenso. Ante esta situación los directivos actuaron con rigidez en las labores de control y supervisión, la organización se hizo más rígida, lo que llevó a que la organización entre en un proceso de deshumanización del trabajo, ante esto el deseo de trabajar declinó. Ante aquella problemática y luego de un profundo análisis para la resolución de los problemas, los directivos optaron por rediseñar los empleos y reestructuras las organizaciones creando un ambiente propicio y adecuado para los trabajadores, en otras palabras, mejorar la calidad de vida laboral.

El termino ' calidad de vida laboral ' tuvo sus orígenes en una serie de conferencias patrocinas al final de los años 60 y comienzos de los 70 por el Ministerio de Trabajo de los EE.UU. y la Fundación FORD. Estas conferencias fueron estimuladas por el entonces ampliamente populares fenómeno de la "alienación del trabajador" simbolizado por las huelgas entre la población activa mayoritariamente joven de la nueva planta de monta de la General Motors, de Ohio. Los asistentes consideraron que el termino iba mas allá de la satisfacción del puesto de trabajo y que incluía unas nociones, como la participación en por lo menos algunos de los momentos de adopción de decisiones, aumento de la autonomía en el trabajo diario, y el rediseño de puestos de trabajo, y sistemas y estructuras de la organización con el objeto de estimular el aprendizaje, promoción y una forma satisfactoria de interés y participación en el trabajo. La CVL [...] alcanza el reconocimiento social e institucional gracias a las actuaciones del «Movimiento de CVL». Las reivindicaciones de este nuevo movimiento parten de la necesidad de humanizar el entorno de trabajo prestando especial atención al desarrollo del factor humano y a la mejora de su calidad de vida. A partir de este momento, el tema de la calidad de vida laboral se populariza tanto en los EE. UU. como en Europa, donde la trayectoria y los matices que adquiere el movimiento se alejan de los planteamientos iniciales ligados a la corriente del Desarrollo Organizacional (DO) , para recibir los influjos del enfoque Sociotécnico y de la Democracia Industrial (DI ). Debido a tales diferencias ideológicas, el estudio de la calidad de vida laboral en Europa se identifica con la corriente de la humanización del trabajo, mientras que en los EE.UU. fiel a sus orígenes mantiene su denominación inicial como movimiento de CVL (Walton, 1973; Davis y Cherns, 1975; Suttle, 1977; Nadler y Lawler , 1983; Delamotte y Takezawa, 1984; entre otros ).(Citado en Segureda y Agullo, 2002)

El estudio de la calidad de vida laboral se ha venido abordando básicamente bajo dos grandes perspectivas teórico-metodológicas: la calidad de vida del entorno de trabajo y la perspectiva de la CVL psicológica (Davis y Cherns, 1975; Taylor, 1978; Elizur y Shye, 1990; González et al., 1996). (Citado en Segura y Agullo). Ambas perspectivas presentan diferencias en cuanto a los objetivos que persiguen en su propósito por mejorar la calidad de la vida en el trabajo, en los aspectos del entorno de trabajo que constituyen su objeto de estudio, en el foco de análisis en el que centran su interés y en el nivel de generalidad de sus análisis de la vida laboral.

La perspectiva de la calidad del entorno de trabajo persigue el objetivo de conseguir mejorar la calidad de vida mediante el logro de los intereses organizacionales. Es así que, el centro de sus análisis será el conjunto de la organización entendida como un sistema, llevando a cabo un nivel de análisis macro, es decir, de los diferentes subsistemas que la integran. 
En cambio, la perspectiva de la calidad de vida laboral psicológica muestra mayor interés por el trabajador, ya que desarrolla un microanálisis de aquellos elementos puntuales que constituyen las distintas situaciones de trabajo en las que participa directamente el individuo. Segureda y Agullo consideran que mientras esta segunda corriente teórica señala la importancia de los aspectos subjetivos de la vida laboral y, por tanto, concede al trabajador un papel destacado, la perspectiva de la calidad del entorno de trabajo subordina tales aspectos a las condiciones de trabajo y a los elementos estructurales de la organización.

Ambos enfoques, a pesar de compartir la meta común de mejorar la calidad de la vida laboral, discrepan en cuanto a los objetivos que persiguen. Según Segura y Agullo, la perspectiva denominada CVL psicológica persigue fundamentalmente la satisfacción, la salud y el bienestar del trabajador anteponiendo los intereses individuales a los de la organización. Sin embargo, la perspectiva de la calidad de vida del entorno de trabajo mantiene la postura contraria: alcanzar una mayor productividad y eficacia organizacionales como paso previo sin el cual no sería posible satisfacer las necesidades y demandas de cada trabajador.

\section{LA CALIDAD DE VIDA LABORAL}

Se torna complicado definir qué es la calidad de Vida Laboral (CVL), ya que este concepto se encuentra íntimamente relacionado con diversos temas del ambiente laboral. Asimismo, existe una gran variedad de disciplinas, enfoques teóricos y áreas de estudio desde las cuales se puede abordar este concepto. Esta serie de factores dificultan la definición de la CVL, e impiden que haya un consenso en cuento a esta.

Atendiendo a la distinta naturaleza de los factores involucrados (objetivos, subjetivos) y a su disposición temporal relativa (antecedentes, consecuentes), puede estudiarse la CVL desde cuatro perspectivas complementarias, lo que vendría a constituir el objeto formal de la disciplina:

- Factores antecedentes objetivos: CVL como realidad de la situación laboral del trabajador: conjunto de estructuras y prácticas organizacionales que resultan relevantes para el bienestar laboral del trabajador.

- Factores antecedentes subjetivos: CVL como adaptación subjetiva a la situación laboral por parte del trabajador: recogería todos aquellos procesos de interpretación y/o actuación que realiza el trabajador sobre su situación laboral, y que afectan asimismo a su bienestar laboral.

- Factores consecuentes objetivos: CVL como ajuste persona - puesto de trabajo: alude al grado de ajuste, correspondencia o concordancia que tiene lugar entre el trabajador y el trabajo que tiene que realizar.

- Factores consecuentes subjetivos: CVL como experiencia subjetiva: se refiere a los distintos elementos componentes y estructura interna del bienestar laboral que experimenta el trabajador en su trabajo.

Como se muestra en la Tabla 1, existe una gran variedad de definiciones de la CVL. En líneas generales, se puede clasificar el repertorio de definiciones existentes en dos grandes bloques dependiendo de la valoración objetiva o subjetiva de la CVL. Para aquellos autores que abordan la Calidad de Vida Laboral desde el punto de vista del trabajador destacan variables como las experiencias 
Isabel Granados P.

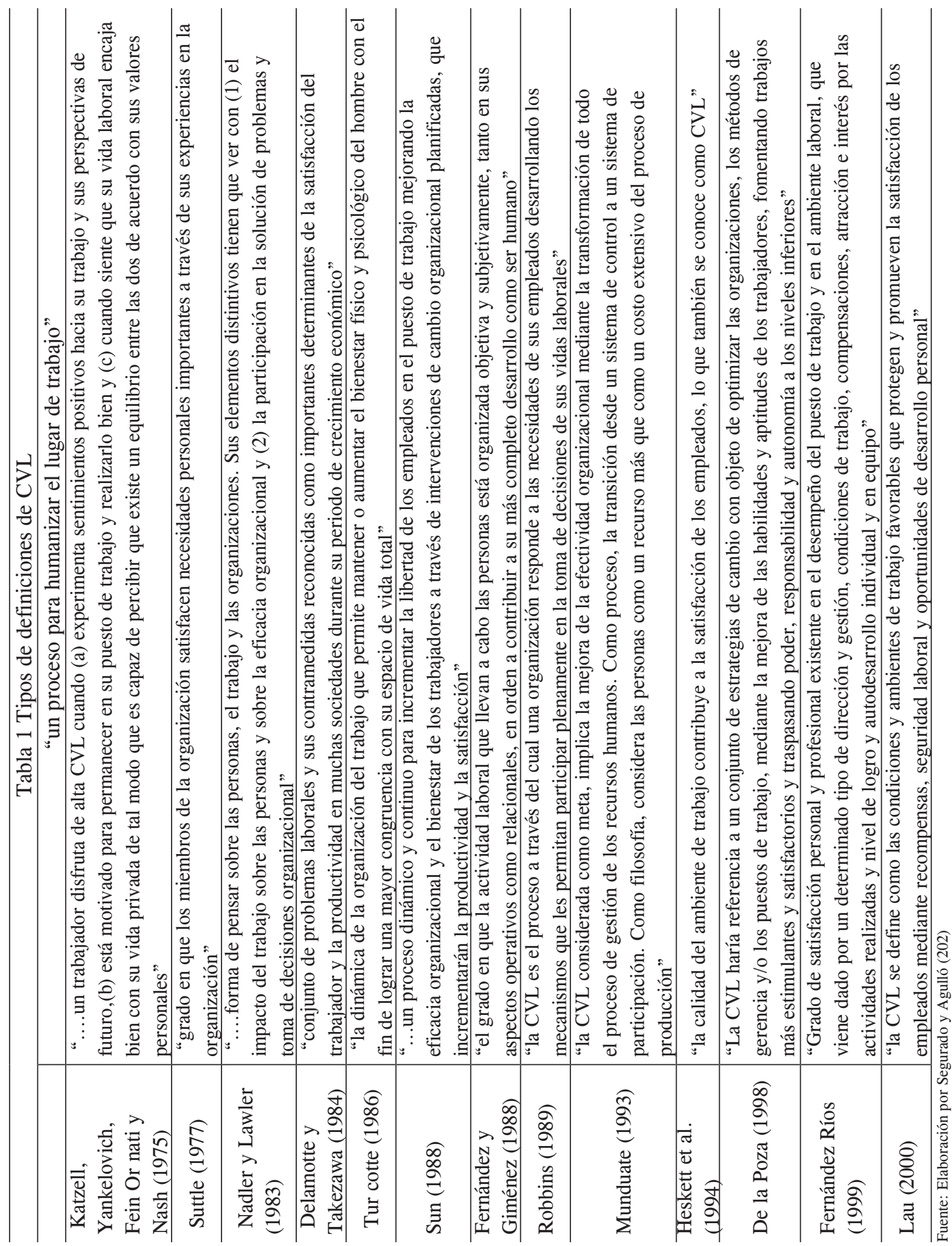


individuales en el ambiente de trabajo, las percepciones, el nivel de motivación y el grado de satisfacción de los individuos. Por otro lado, aquellos autores cuyas definiciones toman como foco de análisis la organización, aluden a la participación, toma de decisiones e implicación de los trabajadores en la dinámica del sistema, las condiciones laborales y aspectos estructurales y estratégicos de la organización. A todo este conjunto más o menos integrado de variables y dimensiones se le denomina CVL: motivación hacia el trabajo; vinculación y necesidad de mantener el equilibrio entre la vida laboral y la vida personal; satisfacción laboral; eficacia y productividad organizacionales; condiciones del entorno socioeconómico; bienestar físico, psicológico y social; relaciones interpersonales; participación del trabajador en el funcionamiento de la organización y en la planificación de sus tareas; autonomía y toma de decisiones de los individuos sobre sus respectivos puestos de trabajo; desarrollo integral del trabajador; estrategias de cambio para conseguir la optimización de la organización; métodos de gerencia de los recursos humanos; condiciones y medio ambiente de trabajo; el trabajador como recurso y no como un costo empresarial / productivo.

\section{DIMENSIONES DE LA CALIDAD DE VIDA LABORAL}

El concepto de calidad de vida laboral es difícil de definir y operacionalizar debido a la complejidad y riqueza de dimensiones que traspasan el limite organizacional y laboral. Este concepto debe de ser considerado multidimensional debido a que abarca diversas condiciones relacionadas con el trabajo que son relevantes para la satisfacción, la motivación y el rendimiento laboral (Casas \& Co., 2002). La CVL como proceso dinámico y continuo en el que la actividad laboral se configura de manera objetiva y subjetiva, tanto en los aspectos operativos como relacionales. Según Casas \& Co. esta perspectiva trata de reconciliar los aspectos del trabajo relacionados con las experiencias humanas y con los objetivos organizacionales.

Las dimensiones que componen a la calidad de vida en el trabajo pueden ser clasificadas en dos grandes grupos; aquellos que se refieren al entorno en el que se realizan las actividades de trabajo (condiciones objetivas) y aquellas que tienen que ver con la experiencia psicológica de los trabajadores. $\square$ Para la mayoría de autores, dada la multidimensionalidad del concepto de CVL, es imposible determinar un conjunto de aspectos que resulten universalmente generalizables $\square$ (Casas \& Co.), sin embargo, es posible mencionar aspectos comunes gran parte de las diversas actividades laborales realizadas por el hombre.

\section{CONDICIONES OBJETIVAS}

Medioambiente físico

En todos los tipos de trabajo que existen, ya sean de producción dentro de una fábrica, trabajo de oficina, trabajo dentro de colegios, hospitales o atención al público, se presentan todo tipo de riesgos laborales: riesgos físicos, químicos, biológicos, entre otros. Las empresas deben de buscar salvaguardar la buena salud de sus colaboradores. 
La fatiga física, relacionada a riesgos posturales, cargas excesivas, etc. son otros de los factores que intervienen directamente en la CVL, y cuya presencia genera la disminución de esta. Como comentan . Dolan \& Co. (1999), existe el síndrome del túnel del carpo bilateral, enfermedad que se caracteriza por el entumecimiento $\mathrm{u}$ hormigueo, dolor y debilidad de las manos y muñecas, el cual es generalmente producido por el trabajo.

Los accidentes laborales constituyen una amplia fuente de insatisfacción laboral. Asi, un accidente como la pérdida de un miembro corporal, de audición o de vista con los más comunes. Generan a su vez grandes pérdidas personales y organizacionales, pudiendo ser estas tan graves que inhabiliten al trabajador de por vida, o se llegue a perder la vida como consecuencia de este.

Falta de espacio físico en el lugar de trabajo o la inadecuación al este, así como el deficiente mobiliario, iluminación, etc. constituyen una dimensión del medio físico que afecta la calidad de vida laboral. Dolan \& Co. (1999) dan un claro ejemplo de esto cuando mencionan el síndrome del edificio de oficinas enfermo. Estos autores manifiestan que este es un conocido malestar en las personas, que se piensa se debe a una mala ventilación, una calidad deficiente del aire y la presencia excesiva de agentes irritantes fuertes en muchos materiales de construcción.

Medioambiente tecnológico

La frustración que genera al trabajador el no contar con los equipos e instrumentos necesarios para realizar el trabajo de manera adecuada constituye una dimensión del medio ambiente tecnológico que afecta de manera negativa a la CVL, ya que genera en el trabajador: ansiedad, falta de motivación, insatisfacción laboral; y a nivel de organización retrasos en la entrega de trabajos o decaimiento de la producción. Todo lo anterior lleva a la falta de efectividad.

La deficiencia en el mantenimiento o suministro de componentes es otra dimensión que genera malestar en los trabajadores, ya que los imposibilita de seguir con sus labores de manera normal. Disminuye su eficiencia, genera estrés, ansiedad y frustración.

Medioambiente contractual

El salario es uno de los aspectos más valorados en el trabajo, aunque su efecto motivador se debe a diversos factores. Al respecto, Casas \& Co. Afirman que es importante tener en cuenta la Teoría de la Equidad de Adams. En esta se explica que las personas distinguen entre las contribuciones que estos aportan en su trabajo y las compensaciones que estos reciben por ellas; así como las que reciben otras personas. Es así como los trabajadores establecen el criterio de equidad, o la falta de este. Como consecuencia, se genera un estado de tensión que impulsa al trabajador a intentar reducir este desequilibrio. Cuando los trabajadores perciben que la relación entre su salario y su rendimiento no es justa se puede producir insatisfacción, absentismo, deseos de abandonar la empresa, bajo rendimiento, falta de dedicación, conflicto de intereses, etc. 
La estabilidad en el puesto de trabajo es un factor importante en la CVL, ya que está demostrada su relación directa con la satisfacción laboral y el compromiso organizacional. Su ausencia genera alteraciones como el estrés, ulceras, ansiedad, depresión, quejas somáticas, migraña, entre otras. Asimismo, la inestabilidad laboral hace que los empleados estén más propensos a recibir sobrecarga laboral, en un intento desesperado por mantener su puesto de trabajo.

Medioambiente productivo

Los horarios de trabajo muy prolongados o los horarios rotativos constituyen un factor importante en la vida del trabajador, las personas que trabajan largos turnos o turnos rotativos experimentan dificultades para mantener sus relaciones sociales y familiares normales. Asimismo, este tipo de horarios supone un impedimento no solo para el esparcimiento y mantenimiento de buenas relaciones familiares y sociales, sino también para la actualización profesional. Del mismo modo, es importante resaltar los problemas de insomnio, fatiga y alteraciones del ritmo circadiano que presentan las trabajadores, lo que afecta de manera directa su CVL.

La sobrecarga laboral ya sea cuantitativa (exceso de actividades a realizar en un determinado periodo de tiempo, o un exceso de horas de trabajo) o cualitativa (excesiva demanda en relación a las competencias, conocimientos y habilidades del trabajador o un gran nivel de responsabilidad en la toma de decisiones) constituye un agente generador de estrés e insatisfacción laboral (Fernandez \& Co., 2000). Es importante reflexionar sobre la situación contraria, la falta de tareas durante la jornada laboral y la asignación de tareas rutinarias y aburridas en relación con las habilidades del trabajador son otras de las dimensiones que afectan la CVL. Los trabajadores que afrontan esta situación se sienten desmotivados, insatisfechos, frustrados y aburridos en el trabajo.

Tanto las oportunidades de promoción y ascenso como las facilidades de formación constituyen un factor motivacional para los trabajadores, el cual incrementa la CVL, debido a que permite un desarrollo personal, mayor autonomía en el trabajo y la posibilidad de realizar tareas más interesantes y significativas. Las oportunidades de promoción y ascenso se encuentran directamente relacionadas con la satisfacción laboral y ayudan a retener a los talentos de la empresa. Estas constituyen, por tanto, una herramienta de gestión de RRHH.

\section{CONDICIONES SUBJETIVAS}

Esfera privada y mundo laboral

Los problemas dentro de la esfera laboral tales como enfermedad de miembros de familia, problemas de tipo afectivo, necesidad de atención extra a los hijos constituyen una dimensión que repercute en la CVL. Ya que repercuten de manera directa en la CV de quien las experimenta. Asimismo, las exigencias del trabajo pueden llevar a los trabajadores a dejar de lado no solo las responsabilidades familiares, sino también las relaciones sociales del trabajador. 
Individuo y actividad profesional

Esta dimensión se relaciona a la utilización y desarrollo de conocimientos, destrezas y habilidades a fin de lograr alcanzar las metas profesionales, lo cual constituye un factor motivador ya que produce sentimientos de logro y de satisfacción con el trabajo (Peiro, 1996). Asimismo, la autonomía y la participación activa en la toma de decisiones son la base de la satisfacción laboral (Casas).

Organización y función directiva

Las reilaciones entre los miembros de los grupos de trabajo constituyen un factor primordial para la salud personal y organizacional (Casas). Se valora mucho la posibilidad de relación e interacción con los compañeros de trabajo. Inclusive, el enfoque de la gestión del conocimiento, resalta la importancia de la interrelación de los trabajadores en la construcción y el compartir del conocimiento. A través de la interrelación con los compañeros, los trabajadores desarrollan no solo sus habilidades sociales, sino también comparten y consolidad conocimientos a través del relato de sus experiencias. Por el contrario, en "Desencadenantes del estrés laboral", Peirò (1999) afirma que la ausencia de contacto con otros trabajadores y la falta de cooperación y apoyo entre compañeros pueden producir un elevado nivel de estrés y tensión entre los miembros del grupo.

La confianza, comunicación espontánea y el apoyo mutuo constituyen un factor importante en la CVL y juegan un rol importantísimo en el éxito del grupo como tal (Rodríguez, 1997). A medida que estas relaciones son vagas o inexistentes, se presentan diversos problemas que se relacionan al trabajo en equipo. Por otro lado, cuanto mayor es la cohesión de los miembros del grupo mayor es la motivación y menor el absentismo.

\section{Beneficios}

La aplicación de programas de mejoramiento de la Calidad de Vida Laboral generan infinidad de beneficios tanto a la empresa como al trabajador, entre los principales podemos observar que muchos estudios demuestran una positiva relación entre las prácticas de innovación en los lugares de trabajo y el éxito empresarial. Los beneficios para la empresa incluyen en diversos aspectos.

Mejora el Funcionamiento Financiero: Investigadores del Business Daily notaron que en 1999 Home Depot, se caracterizaba por "una cultura basada en la libertad", en la cual los empleados son animados a tomar riesgos en las decisiones de sus negocios, con ello las ganancias crecieron en un promedio anual del $24 \%$. Asimismo, el libro escrito en 1998 por John Case, The Open Book Experience, informa que las compañías con un manejo abierto, un sistema participativo que incorpore la transparencia empresarial en el poder de decisión del empleado y una compartida apuesta al éxito financiero en la compañía, experimentan significativos aumentos en la productividad, eficiencia, ventas y beneficios. Case afirma que las compañías que adoptan el estilo de dirección Open Book tempranamente en su existencia, como Whole Foods, AES Corp. Y PSS/World Medical, han crecido rápidamente para convertirse en industrias líderes. Del mismo modo es 
importante mencionar el estudio de la Fundación Ford conducido con Xerox, Corning y Tandem Computadores que encontraron una positiva correlación entre las compañías con políticas de calidad de vida laboral y su actuación financiera.

Incrementa el Valor de Mercado. Variados estudios han demostrado que los beneficios derivados de programas y estrategias efectivos en lugares de trabajo - y los costos por no hacerlo- están unidos al valor del stock de la compañía. Así, en 1998, un estudio realizado por Ernst \& Young a 275 gerentes, detectó que el 35\% de todas las decisiones sobre inversión son manejadas por factores no financieros. La habilidad de atraer o retener a empleados talentosos está rankeado entre los 5 factores de inversión usados para escoger stocks.

Incrementa la productividad. En 1998, la empresa Fleet Financial Group rediseño las labores y obligaciones en dos de sus sitios de trabajo para acomodar la vida de laboral de los empleados y sus necesidades, y se encontró que el estrés declinó, el cambio de un lugar a otro se redujo y las metas de producción fueron cumplidas o excedidas. Asimismo, la revista, "Working Mother " informo en 1997 que el Centro de Procesos Financieros, Hewlett-Packard doblo su productividad cuando algunos de los trabajadores comenzaron a utilizar semanas de trabajo comprimidas.

Reduce los Costos Operativos. El estudio de la Conferencia 2000, entre 200 ejecutivos de 158 compañías encontró que un gran número de compañías multinacionales reportó un gran ahorro e incremento en su funcionamiento, después de implementar nuevas formas de trabajo que incentivaban a los empleados a crear y compartir conocimientos. Por ejemplo, BP Amoco y Ford Motor Company han ahorrado cada una $\$ 600$ millones de dólares a través de sus programas de manejo de conocimiento. En 1999, "HR Focus " reportó que la oficina de Retención de Ernst \& Young's, diseño programas que incrementaron el acceso de los trabajadores a la dirección. Estos programas ahorraron a la firma alrededor de \$ 21 millones de dólares.

Mejora la Habilidad para Retener y Atraer a los Mejores Empleados. Las estrategias para conciliar Trabajo y Vida Personal, se han convertido en aspectos muy atractivos y significativos, especialmente, entre profesionales jóvenes y trabajadores especializados. En 1997 se realizó un estudio a dos mil administradores en 4 compañías y éste arrojó que más del $75 \%$ de ellos, llegó a la conclusión que ofrecer flexibilidad laboral incentivaba la retención de empleados. En suma, el $92 \%$ de los empleados part-time incrementaron su bienestar y un 53\% reportó un incremento de confianza hacia la compañía.

Fortalece la Confianza y Lealtad de los Empleados. Algunos estudios sugieren que el nivel de confianza de empleados esta directamente relacionado con la respuesta de la compañía a sus necesidades. Así, una medición, realizada en 1999, apoyada en la información del Instituto Walker Hudson hecha a dos mil empleados en 48 estados (USA) detectó que los factores que tienen mayor influencia en la calidad de vida laboral son: transparencia, preocupación por el empleado, confianza, reputación de la empresa, trabajo y recursos para el trabajador. Del 
mismo modo, en 1998 un estudio de WFD en colaboración con MORI realizado en el Reino Unido, indicó que 9 de 10 trabajadores declaraban que la habilidad para balancear el trabajo con su vida personal era el factor clave para determinar su confianza hacia su empresa.

Reduce el Ausentismo: Estudios muestran de que a mayor compromiso de la empresa con sus empleados, hay menor tasa de ausentismo. En Florida (USA) el Equipo Aladdin, realizó una reducción de un 50\% en ausentismo y aumentó la productividad en un $10 \%$, después de implementar una jornada flexible de 4.5 días a la semana.

Fortalece la Reputación: Los programas de calidad de vida laboral en la empresa pueden fortalecer la reputación de la compañía entre sus empleados, consumidores, proveedores e inversionistas y también con la comunidad en la cual operan. En 1998 un estudio de la Corporación Cultural realizado por el grupo Hays, en el que se estudiaron 10 compañías del listado de "Las Compañías más Admiradas" de la revista Fortune, encontró que "las compañías más admiradas son las que han decidido poner énfasis en el trabajo de equipo, focus groups, en la transparencia en el tratamiento a sus trabajadores, en la iniciativa y las innovaciones".

\section{CONCLUSIONES}

Tratar y entender la calidad de vida laboral bajo una aproximación psicosocial significa, de entrada, tener en cuenta los procesos psicosociales básicos que se originan en el entorno de trabajo, considerando la interdependencia entre los aspectos psicológicos y sociales a partir de los que se construye y configura la realidad laboral. Dicho de otro modo, la CVL es el resultado de la actuación de un individuo socializado por, para y en el trabajo (en un entorno organizacional), un individuo que se comporta y se relaciona en un contexto significativo y determinado, su entorno socio-laboral específico (Blanco, 1985; Agulló, 1997; Fernández Ríos, 1999).

El se debe tratar de abordar el estudio de la calidad de vida en el trabajo desde un enfoque caracterizado por un carácter integrador (psicosocial: no podemos olvidar la pretensión transdisciplinar de la Psicología Social) y tiene como propósito comprenderla de forma holística, sistémica, integral y contextualizada. La naturaleza multidimensional e interactiva del constructo obliga a tener en cuenta los siguientes principios y criterios definitorios para delimitarla conceptualmente:

Dado que la calidad de vida laboral engloba múltiples aspectos relacionados con el medio laboral (y todo lo que éste supone y entraña) es necesario considerar al referirnos a ella globalmente que se compone de dos grandes dimensiones que, por separado, únicamente ofrecerían una visión parcial y sesgada de la vida en el trabajo. La dimensión objetiva y la dimensión subjetiva de la calidad de vida laboral se refieren, por un lado, a aquellos elementos derivados de las condiciones de trabajo, al desempeño del puesto y en general al contexto organizacional; $y$, por 
otro, a los procesos psicosociales a través de los cuales el trabajador experimenta dicho entorno de trabajo.

La vida laboral se ve afectada no solamente por factores intrínsecos al entorno de trabajo, sino también por todo lo que acontece en otras áreas extralaborales que conforman la cotidianidad de los individuos (la familia, los amigos, la red de contactos, el ocio y el tiempo libre). Esta interdependencia da lugar a una estrecha vinculación entre lo laboral y lo extralaboral que, además de condicionar la calidad de vida laboral, también determina los modos de vida y el estatus social de los individuos.

La calidad de vida en el trabajo no se refiere únicamente a las condiciones físicas del lugar de trabajo ni tampoco a la subjetividad de un colectivo de trabajadores, sino que se define y se materializa en la relación dialéctica que el individuo mantiene con su ambiente de trabajo. Fruto de esta interacción el individuo construye y modifica su lugar de trabajo y es este carácter interactivo el que le permite desempeñar su rol como trabajador (que, por ende, afectará a su papel y estatus como agente social).

El carácter interactivo de la calidad de vida laboral la convierte en una totalidad integrada (holística y sistémica) de elementos interdependientes e interrelacionados que, articulados entre sí, conforman un todo indisociable, núcleo de estudio y análisis.

\section{REFERENCIAS BIBLIOGRÁFICAS}

Agulló, E. (1997). Naturaleza psicosocial del trabajo: significado, centralidad, socialización, futuro». En Agulló, E. Jóvenes, trabajo e identidad. Universidad de Oviedo: Servicio de Publicaciones.

Blanco, A. (1985). La calidad de vida: supuestos psicosociales. En Morales, J.F., Blanco, A., Huici, C. y Fenández, J.M. Psicología Social Aplicada. Bilbao: DDB

Casas J. \& Co. (2002) Dimensiones y medición de la calidad de vida laboral en profesionales sanitarios Revista de administración sanitaria, Vol VI - No. 23.

Dolan S. \& Co. (1999) La gestión de los recursos humanos Madrid: McGraw-Hill

Fernández Ríos, M. (1999). Diccionario de RRHH. Organización y dirección. Madrid: Díaz de Santos.

Fernandez \& Co. (2000) Satisfacción de los profesionales de Atención Primaria del Área 10 del INSALUD de Madrid Revista Esp Salud, Vol 74.

Loli A. y Cuba E. (2007). Autoestima y compromiso organizacional en trabajadores de una universidad pública de provincias Revista de Investigación en Psicología, Vol 10 - No. 1

Peiró,J.y Co.(1996): Tratado de psicología del trabajo. Madrid: Síntesis

Peiró,J.(1999) Desencadenantes del estrés laboral. Madrid: Piramide. 
Peiró, P. (1998). Cuestionario de Satisfacción Laboral S20/23. Psicología de la seguridad laboral. Consultado el 15 de diciembre del 2010 en http://www.uv.es/meliajl/ Research/Cuest_Satisf/S20_23.PDF

Segurado, A. y Agulló, E. (2002) Calidad de vida laboral: hacia un enfoque integrador desde la Psicología Social Psicothema, Vol 14 - No.4

Rodrigues, JM \& Co. (1997). El reto del trabajo en equipo. Biblioteca IESE de Gestión de Empresas Barcelona, Ediciones Folio. S.A.

Turcotte, P.R. (1985). Calidad de vida en el trabajo. Madrid: Trillas.

Referencias electronicas

Education Fund, Business for Social Responsibility:

http://www.accionrse.cl/app01/home/calidadLaboral.html 